\title{
MUDANÇAS CLIMÁTICAS E O SETOR HIDROELÉTRICO BRASILEIRO: UMA ANÁLISE COM BASE EM MODELOS DO IPCC-AR5
}

CLIMATE CHANGE AND BRAZILIAN HYDROPOWER SECTOR: AN ANALYSIS BASED ON GLOBAL MODELS FROM IPCC-AR5

\section{Cleiton da Silva Silveira}

Professor adjunto do Instituto de Engenharia e Desenvolvimento Sustentável, Universidade da Integração Internacional da Lusofonia Afro-brasileira (UNILAB) Redenção (CE), Brasil.

\section{Francisco de Assis}

\section{Souza Filho}

Professor adjunto do Departamento de Engenharia Hidráulica e Ambiental (DEHA), Universidade Federal do Ceará (UFC) Fortaleza (CE), Brasil.

\section{Francisco das Chagas} Vasconcelos Júnior

Pesquisador, Fundação Cearense de Meteorologia e Recursos Hídricos (FUNCEME) - Fortaleza (CE), Brasil.

\section{Luiz Martins de} Araújo Júnior

Doutorando em Engenharia Civil, UFC - Fortaleza (CE), Brasil.

\section{Samuellson Lopes Cabral}

Tecnologista, Centro Nacional de Monitoramento e Alertas de Desastres Naturais (CEMADEN) São José dos Campos (SP), Brasil.

\section{Endereço para correspondência:}

Cleiton da Silva Silveira -

Avenida da Abolição, 3-CentroCEP 62790-000-Redenção (CE), BrasilE-mail: cleitonsilveira@unilab.edu.br

Recebido: 29/11/2016

Aceito: 30/11/2017

\section{RESUMO}

O objetivo deste trabalho foi analisar o impacto das mudanças climáticas nas vazões dos postos do setor elétrico brasileiro utilizando seis modelos globais do Intergovernmental Panel on Climate Change - Fifth Assessment Report (IPCC-AR5) para os cenários RCP4.5 e RCP8.5 no período de 2011 a 2098. As vazões de 21 postos representativos do Operador Nacional do Sistema (ONS) do Sistema Interligado Nacional (SIN) foram geradas por meio do modelo hidrológico Soil Moisture Account Procedure (SMAP), enquanto as vazões dos demais postos que compõem o SIN foram obtidas por regressões lineares. Foram analisadas as anomalias das vazões médias anuais e a tendência no período de 2011 a 2098. Os modelos do IPCC-AR5 mostraram que há maior possibilidade de redução nas vazões anuais na maior parte do Brasil, exceto para a Região Sul, onde os modelos mostram aumentos superiores a 5\% no período de 2040 a 2069.

Palavras-chave: vazão; setor hidroelétrico brasileiro; mudanças climáticas.

\section{ABSTRACT}

The objective of this study was to assess the climate change impacts on the annual streamflows of the brazilian hydropower sector by using six global models from Intergovernmental Panel on Climate Change - Fifth Assessment Report (IPCC-AR5) for RCP4.5 and RCP8.5 scenarios from 2011 and 2098. Streamflow from 21 relevant National Electric System Operator (ONS) stations from National Interconnected System (NIS) were generated through the hydrological model Soil Moisture Account Procedure (SMAP), while the streamflow from other stations that compose the NIS were obtained by linear regression. Annual mean streamflow anomalies and trend were analyzed in the period from 2011 to 2098 . The IPCC-AR5 models showed that there is a greater possibility of reduction in annual streamflows in most of Brazil, except for the southern, where the models showed increases more than $5 \%$ during 2040-2069.

Keywords: streamflow; hydropower sector; climate change. 


\section{INTRODUÇÃO}

As mudanças climáticas podem produzir grandes impactos sobre os recursos hídricos. É possível que o aumento da temperatura média global observado nas últimas décadas cause alterações no ciclo hidrológico, por meio de modificações dos padrões de precipitação e evapotranspiração capazes de impactar diretamente a umidade do solo, a reserva subterrânea e a geração do escoamento superficial (BATES et al., 2008). Esses aspectos, associados ao aumento da demanda por água, estão projetados para as próximas décadas, principalmente em razão do crescimento populacional e do aumento da riqueza; regionalmente, poderão exercer grande pressão nos hidrossistemas brasileiros.

O Painel Intergovernamental sobre Mudança do Clima (IPCC) garante que mudanças climáticas impõem uma grande ameaça ao desenvolvimento sustentável, por afetar de forma direta e indireta grande parte da população, sua saúde, os recursos hídricos, as infraestruturas urbana e rural, as zonas costeiras, as florestas e a biodiversidade, bem como os setores econômicos como agricultura, pesca, produção florestal, geração de energia, indústrias - e suas cadeias (CHIEW et al., 2009). O IPCC aponta impactos de grande magnitude sobre a América do Sul, especialmente quanto a recursos hídricos e setores econômicos relacionados, impondo a necessidade de formular medidas de adaptação, com vistas a gerenciar riscos climáticos. Dessa forma, torna-se fundamental a elaboração de subsídios ao planejamento nacional de longo prazo que incorporem a mudança do clima.

A matriz energética brasileira é basicamente constituída por energias consideradas limpas, principalmente provenientes de hidroelétricas, extremamente dependente do regime de vazões nos rios em que estão localizados os empreendimentos. Qualquer modificação nos padrões do ciclo hidrológico pode representar impactos significativos na geração de energia, tornando o sistema mais vulnerável (PRADO JR. et al., 2016; SOITO; FREITAS, 2011).

A variação do escoamento nos rios é influenciada por diversos fatores, entre os quais se destacam a precipitação ocorrida na bacia de contribuição e as mudanças no uso e na ocupação do solo. Portanto, a geração de hidroeletricidade no Brasil exige uma análise sobre o regime fluvial e seus padrões de variação temporal, dado o significativo impacto que essas variações podem produzir na oferta de energia e, consequentemente, em toda a economia brasileira (ALVES et al., 2013). Diante disso, existe uma demanda pelo Estado e pelas empresas privadas por informações climáticas para tomada de decisão a nível regional/local de médio e longo prazos. Informações de variabilidade e mudanças climáticas de qualidade podem tornar o planejamento energético mais eficaz e minimizar os potenciais impactos acerca da disponibilidade desse recurso (BANCO MUNDIAL, 2010).

As mudanças e a variabilidade climáticas têm sido alvo de discussões e pesquisas científicas em todo o mundo, com vistas ao entendimento de sua ocorrência (IPCC, 2007a; 2007b; NOBRE, 2005; SILVEIRA et al.,2013a; 2013b; 2013c; SILVEIRA et al., 2012; SOUZA FILHO, 2003; MARENGO; SOARES, 2005; MARENGO; VALVERDE, 2007;ARNEEL, 2004; VAN VILET et al., 2012; VAN VILET et al., 2016). Os impactos no escoamento de água superficial e na recarga de água subterrânea variam dependendo da região e do cenário climático considerado (IPCC, 2014), mas relacionam-se, principalmente, com as alterações projetadas para a precipitação (IPCC, 2001; KROL et al., 2006).

Muitos estudos foram realizados com o objetivo de verificar o impacto da mudança do clima nos recursos hídricos da América do Sul. Milly et al. (2005) afirmam que há concordância nas projeções para a metade do século XXI, mostrando aumento de vazão sobre a Bacia do Paraná-Prata e redução nas bacias do leste da Amazônia e do Nordeste do Brasil, baseados em modelos do IPCC-AR4 (quarto relatório do Painel Intergovernamental de Mudanças Climáticas) no cenário A1B. Tomasella et al. (2009) acoplaram um modelo hidrológico para grandes bacias a um modelo regional climático forçado com modelo global HadCM3 para o cenário A1B do IPCC-AR4. Esse modelo regional climático revelou projeções de redução de $30 \%$ na vazão mensal sobre a Bacia do Rio Tocantins, indicando que maiores reduções podem ocorrer durante o período de estiagem. Silveira et al. (2014) analisaram o impacto nas vazões nas bacias do sistema elétrico brasileiro com base em projeções dos modelos globais do IPCC-AR4, forçando modelos hidrológicos chuva-vazão para os cenários $A 1 B, B 2$ e A2. Os resultados mostraram grande divergência nos valores das vazões anuais para o Nordeste 
do Brasil, especialmente na Bacia do Xingó, associada a reduções nas vazões no Setor Sudeste do país, em torno de $5 \%$ em Furnas.

Van Vilet et al. (2012) e Van Vilet et al.(2016) mostram que sistemas hidrotérmicos são muito vulneráveis às mudanças climáticas, destacam a dependência daenergia gerada por hidroelétricas em relação à disponibilidade de água e sinalizam que o aquecimento global pode afetar o resfriamento da água das termoelétricas, diminuindo sua eficiência. Os autores afiançam que é necessário um planejamento energético com enfoque mais forte na adaptação do setor elétrico, além de mitigação de impactos, recomendando que ações sejam tomadas para prover a segurança hídrica e energética nas próximas décadas.

Sistemas energéticos como o brasileiro estão sujeitos a impactos advindos da variabilidade e das mudanças no clima, tanto na produção de energia, em suas diversas formas, quanto no seu consumo - visto que o planejamento e a geração eletroenergética do Sistema Interligado Nacional (SIN) apresentam correlação com os estoques de água existentes nos reservatórios das usinas hidrelétricas e das suas afluências. Sobretudo, em períodos críticos, com reservatórios com baixos volumes, são acionadas termoelétricas de emergência para suprir a demanda energética do país. As térmicas no Brasil acabam por funcionar como reservatórios virtuais ao proporcionar segurança de abastecimento quando os reservatórios estão vazios e ao aliviar a necessidade de estocar água para lidar com a incerteza das afluências.

As mudanças climáticas representam um desafio para a gestão de recursos hídricos e energéticos do Brasil. Prado Jr. et al. (2016) reforçam que a política de crescimento energético do país precisa ser revista em busca de garantir a segurança energética a longo prazo perante as mudanças climáticas e sugerem que esforços precisam ser concentrados em melhoria da eficiência energética, além de investimentos em energia eólica e solar. Prado Jr.et al. (2016) destacam ainda que, para garantir o crescimento econômico do país, é necessária a construção de alguns empreendimentos hidroelétricos, entretanto ressaltam que alguns, especialmente na Região Norte do país, precisam ser cancelados ou adiados, para evitar e/ou minimizar danos socioeconômicos e ambientais na região.

Diante disso, o objetivo deste trabalho foi analisar o impacto das mudanças climáticas nas vazões dos postos do setor elétrico brasileiro utilizando seis modelos do IPCC-AR5 para os cenários RCP4.5 e RCP8.5 no período de 2011 a 2098.

\section{METODOLOGIA}

Esta seção mostra os cenários do IPCC-AR5 utilizados, além dos procedimentos para a obtenção das vazões para os aproveitamentos hidroelétricos do SIN.

A metodologia de obtenção das vazões divide-se, basicamente, em cinco etapas:

- A primeira etapa consiste em escolher as bacias representativas que contemplam espacialmente a maior parte do SIN. Conforme esse critério, foram selecionadas 21 bacias, dispostas como a Figura 1. Em seguida, são selecionados os dados de estações do Instituto Nacional de Meteorologia (INMET) no período de 1992 a 2007 (precipitação, insolação, temperatura, umidade), para calibração dos parâmetros do modelo hidrológico Soil Moisture Accounting Procedure (SMAP) (LOPES et al., 1981). Para tanto, é utilizado um procedimento de otimização objetivo com base no coeficiente de Nash-Sutcliffe, ao comparar a série de vazões obtidas pelo SMAP com a série dispo- nibilizada pelo Operador Nacional do Sistema (ONS). Mais detalhes sobre o modelo SMAP podem ser encontrados no subtópico "Modelo hidrológico";

- A segunda etapa fundamenta-se na obtenção das precipitações dos modelos globais do IPCC-AR5 para os cenários Historical, RCP4.5 e RCP8.5 para 21 bacias de interesse, conforme a Figura 1, para posterior correção estatística para remoção de viés usando a função de distribuição gama. Essa correção utilizou como base de dados de precipitação mensal o Climate Research Unit (CRU) (NEW et al.,2002). Para período histórico, usou-se a série mensal de 1950 a 1999, enquanto para as projeções se utilizoua série de 2010 a 2098. Mais detalhes sobre os modelos globais empregados estão no subtópico "Modelos do IPCC-AR5";

- A terceira etapa pauta-se em obter as evapotranspirações potenciais por meio dos modelos globais do IPCC-AR5 para os cenários Historical, RCP4.5 
e RCP8.5, usando o método de Pennan-Motheih (ALLEN et al., 1998). Para tanto, são usadas como dados de entrada as temperaturas máxima, mínima e média dos modelos globais do IPCC-AR5;

- Na quarta etapa, obtêm-se as vazões usando modelo hidrológico SMAP (LOPES et al., 1981; SOUZA FILHO; PORTO, 2003) em 21 postos, tendo como dados de entrada as evapotranspirações estimadas e as precipitações com viés removido;

- A quinta etapa consiste em obter as vazões estimadas com base nos dados dos modelos globais para os postos que não possuem o modelo hidrológico calibrado.Para isso, são utilizadas regres- sões mensais a partir dos postos que possuem o SMAP calibrado.

Para realizar esse procedimento, são utilizadas as séries mensais de vazões naturalizadas disponibilizadas pelo ONS no período de 1931 a 2009. As séries de vazões do ONS são divididas em dois grupos: um com 21 postos e outro com 167, sendo a vazão mensal dos 21 postos usada como preditora da vazão dos demais postos. Logo, obtêm-se os parâmetros da regressão dos 167 postos usando stepwise.

Com as vazões dos modelos globais do IPCC-AR5 estimadas pelo SMAP para as 21 bacias e com os parâmetros gerados para os outros 167 postos, são estimadas as vazões dos 188 postos que compõem o SIN.

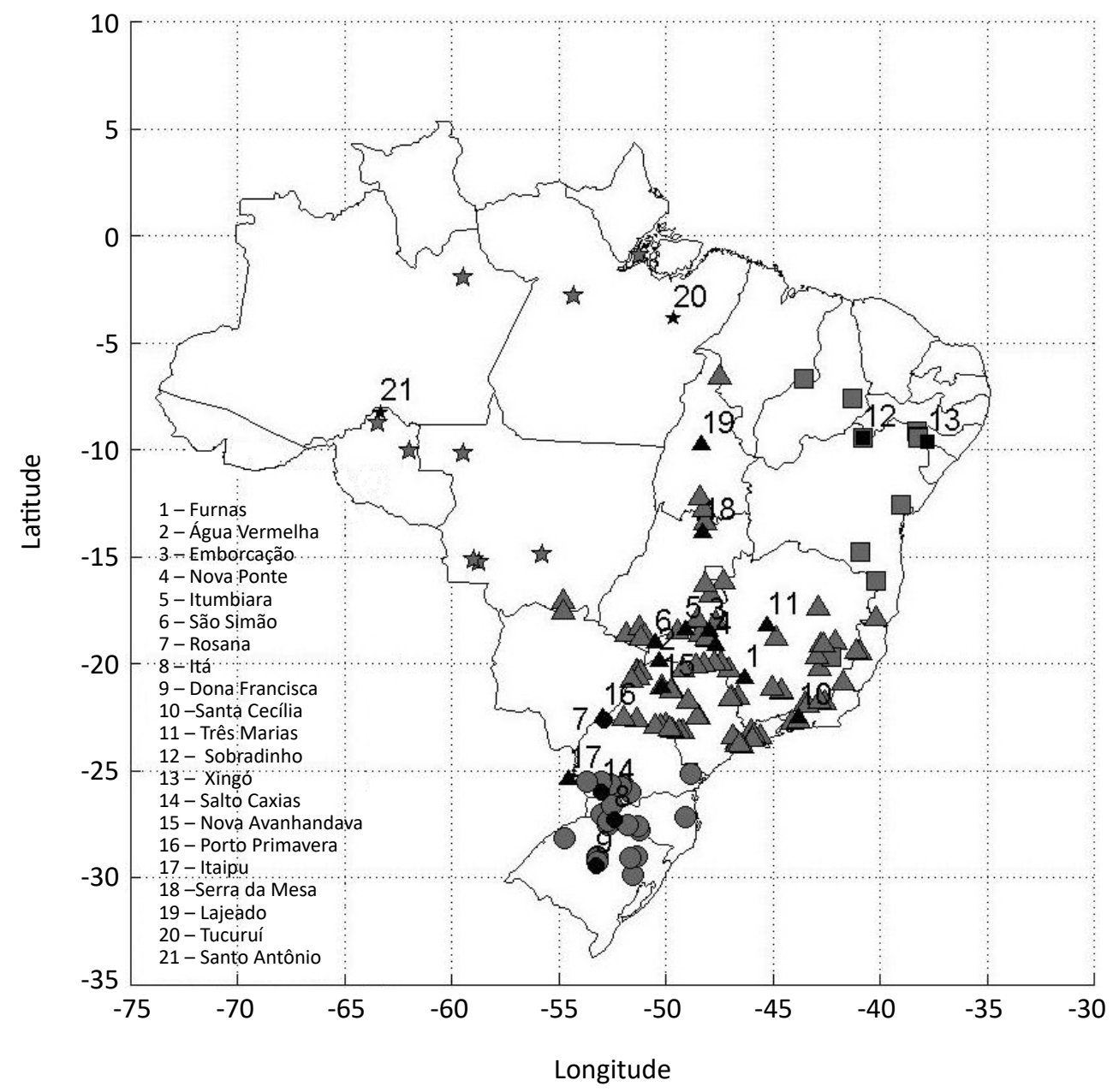

Figura 1 - Aproveitamentos hidroelétricos do Sistema Interligado Nacional. Os postos numerados representam aqueles que foram gerados a partir do uso do modelo hidrológico, enquanto os demais

representam os postos cujas vazões foram geradas por meio de regressões lineares. A estrela representa o subsistema Norte; o quadrado, o Nordeste; o triângulo, o Sudeste; e o círculo, o Sul. 


\section{O Sistema Interligado Nacional}

O SIN responde pela produção e transmissão de energia elétrica do Brasil. É um sistema hidrotérmico de grande porte com predominância de usinas hidroelétricas.

Esse sistema possui grande variabilidade espacial por conta das diversas localizações dos postos no país, sofrendo influência de vários fenômenos meteorológicos e indicando comportamentos sazonais distintos para as bacias que compõem o sistema. A fim de aproveitar ao máximo os recursos energéticos existentes do SIN e a sazonalidade hidrológica própria de cada região, o sistema é dividido em quatro subsistemas: Setor Sudeste/Centro-Oeste, Setor Sul, Setor Norte e Setor Nordeste. Esses subsistemas são interligados por uma extensa malha de transmissão que possibilita a transferência de excedentes energéticos e permite a otimização dos estoques armazenados nos reservatórios das usinas hidroelétricas.

A Região Norte produz $8 \%$ da energia nacional em GWh e demanda 7\%, a Região Nordeste produz $11 \%$ e demanda $18 \%$, a Região Sul produz $18 \%$ e demanda $16 \%$, e a Região Sudeste/Centro-Oeste produz $47 \%$ e demanda $61 \%$.

A previsão de vazões e a geração de cenários de afluências definidas em ONS $(2009,2012 b)$ estabelecem os processos para a previsão de vazões mensais, semanais e diárias e para a geração de cenários de afluências naturais médias mensais utilizadas na elaboração do Programa Mensal da Operação Energética (PMO).

\section{Modelos do IPCC-AR5}

Os dados provenientes do IPCC são resultados de simulações de modelos atmosféricos globais de centros de pesquisa que contribuem para a confecção do relatório do IPCC-AR5 (Quadro 1). Os modelos possuem como forçantes as concentrações de gases de efeito estufa durante o século $X X$ e as estimativas de concentração no século XXI.

Como parte da fase preparatória para o desenvolvimento dos novos cenários para o AR5, foram criados os chamados representative concentration pathways (RCPs), que servem como entrada para modelagem climática e química atmosférica nos experimentos numéricos do Coupled Model Intercomparison Project Phase 5 (CMIP5). Os RCPs recebem seus nomes a partir dos níveis das forçantes radiativas, conforme relatado pelas equipes de modelagem
Em razão das metodologias e dos critérios atualmente adotados na previsão de vazões, o ONS pode não utilizar a disponibilidade de vazões mensais para alguns locais de aproveitamento em operação. Para tanto, adota-se, em geral, a realização de previsão de vazões para um subconjunto de aproveitamentos de cada bacia, denominados de postos-base. No restante dos locais de aproveitamento, as vazões são previstas por meio de regressões lineares mensais com base nos dados previstos nos postos-base para complementar as previsões de vazões para todo o SIN (ONS, 2012b). O ONS trabalha atualmente com o total de 88 postos-base, representativo dos diversos regimes hidrográficos regionais encontrados em território brasileiro.

Neste trabalho foram utilizados 21 postos-base para a calibração do modelo SMAP, enquanto as vazões dos demais postos foram obtidas por meio de regressões, conforme Figura 1.

Até o fim de 2010 o SIN contava com 206 séries de vazões naturais devidamente divididas em 185 pontos de aproveitamento no sistema, sendo 161 postos naturais, 3 postos artificiais e 21 postos naturais/artificiais. Dos 185 locais de aproveitamento, 169 são postos em operação e 16 postos em expansão com horizonte de projeto até 2015 (ONS, 2012a).

elaboradoras de cada RCP. Assim, RCP-X implica um cenário no qual a forçante radiativa de estabilização ou de pico ao final do século XXI corresponde a X W. $\mathrm{m}^{-2}$. Neste trabaIho foram usados os cenários RCP4.5 e RCP8.5 para análise das projeções do século XXI e três modelos globais do IPCC-AR5, conforme Quadro 1.

O cenário RCP4.5 pressupõe que a forçante radiativa estabilizará pouco depois de 2100 , sem ultrapassar o nível de radiação a longo prazo, de $4,5 \mathrm{~W} / \mathrm{m}^{2}$. Essa projeção é consistente com a estabilização da demanda energética mundial, programas de reflorestamento fortes e políticas climáticas rigorosas. Além disso, sugere a estabilização das emissões de metano associada a um leve aumento das emissões de dióxido de carbono $\left(\mathrm{CO}_{2}\right)$ 
até 2040 , até atingir o valor-alvo de $650 \mathrm{ppm}$ de $\mathrm{CO}_{2}$, equivalente ao da segunda metade do século XXI.

O cenário RCP8.5 sugere crescimento contínuo da população associada ao desenvolvimento tecnológico lento,

\section{Modelo hidrológico}

O modelo chuva-vazão SMAP (LOPES et al., 1981) é do tipo conceitual, determinístico e de estrutura concentrada. Pertence à grande família dos modelos hidrológicos de cálculo de umidade do solo. Sua estrutura é relativamente simples, cujos parâmetros são relacionados com parâmetros físicos médios da bacia.

O SMAP, em sua versão mensal, utiliza em seu esquema conceitual dois reservatórios lineares, representando o solo (camada superior) e o aquífero. A cada evento de precipitação $(P)$ é realizado um balanço de massa. Uma parcela de Pé transferida como escoamento superficial $\left(\mathrm{E}_{\mathrm{s}}\right)$. Esse cálculo é feito por meio da equação do Soil Conservation Service (SCS) para escoamento superficial. A lâmina restante da precipitação subtraída do escoamento superficial $\left(P-E_{S}\right)$ sofre perda por evaporação em nível de evaporação potencial $\left(E_{p}\right)$. Logo, a lâmina remanescente $\left(P-E_{s}-E_{p}\right)$ é adicionada a um reservatório que representa a camada superior do solo. Neste, a umidade é atualizada ao longo do tempo por meio das perdas por evapotranspiração real $\left(E_{r}\right)$, que dependem do nível do reservatório $\left(\mathrm{R}_{\text {solo }}\right)$ e da capacidade de saturação do solo $\left(\right.$ Cap $\left._{\text {sat }}\right)$. Outra saída desse reservatório é a recarga

\section{Obtenção da evapotranspiração}

Para o cálculo da evapotranspiração de referência $\left(E_{p}\right)$, é usado o método de Penman-Monteith (ALLEN et al., 1998; SILVEIRA et al., 2014). Segundo esse método, a $E_{p}$ é dada conforme a Equação 1: resultando em acentuadas emissões de $\mathrm{CO}_{2}$. Esse cenário é considerado o mais pessimista para o século XXI em termos de emissões de gases do efeito estufa, sendo consistente com nenhuma mudança política para reduzir as emissões e forte dependência de combustíveis fósseis.

(Rec) no reservatório subterrâneo $\left(R_{\text {sub }}\right)$, em que é utilizado o conceito de capacidade de campo (Cap) para determiná-la. Esse segundo reservatório também é linear e o nível de água existente $\left(\mathrm{R}_{\text {sub }}\right)$ é deplecionado a uma taxa constante de recessão do escoamento de base $(K)$, resultando em escoamento de base (Eb). A soma do $E_{s}$ e $E_{b}$ fornece a vazão no ponto de controle da bacia.

O SMAP mensal possui quatro parâmetros: $\mathrm{Cap}_{\mathrm{sat}}$; parâmetro que controla o escoamento superficial (PES); coeficiente de recarga, parâmetro relacionado com a permeabilidade na zona não saturada do solo (Crec); e taxa de deplecionamento $\mathrm{K}$ do nível $\mathrm{R}_{\text {sub' }}$ que gera o escoamento de base (Eb). Outras duas variáveis de estado precisam ter seus valores inicializados: taxa de umidade do solo inicial $\left(\mathrm{TU}_{\text {in }}\right)$,que determina o nível inicial do $\mathrm{R}_{\text {solo }}$; e valor do escoamento de base inicial $\left(E B_{\text {in }}\right)$, que define o valor inicial do $R_{\text {sub }}$.

A calibração foi efetuada para o período de setembro de 1997 a agosto de 2002, e a validação para setembro de 2002 a agosto de 2007.Os parâmetros obtidos podem ser vistos em Silveira et al. (2014).

$$
E_{p}=\frac{0,408 \Delta\left(R_{n}-G\right)+\gamma \frac{900}{T+273} u_{2}\left(e_{s}-e_{a}\right)}{\Delta+\gamma\left(1+0,34 u_{2}\right)}
$$

Quadro 1 - Modelos do IPCC-AR5 considerados.

\begin{tabular}{|l|r|r}
\hline Modelos & $\begin{array}{c}\text { Instituição ou agência; país } \\
\text { BCC-CSM1-1 }\end{array}$ \\
\hline $\begin{array}{l}\text { BNU-ESM } \\
\text { CESM1-BGC }\end{array}$ & College of Global Change and Earth System Science, Beijing Normal University; China \\
\hline CSIRO-MK3-6-0 & Community Earth System Model Contributors; Estados Unidos \\
\hline HadGEM2-ES & $\begin{array}{r}\text { Commonwealth Scientific and Industrial Research Organization in collaboration } \\
\text { with Queensland Climate Change Centre of Excellence; Austrália }\end{array}$ \\
\hline MIROC5 & Met Office Hadley Centre; Reino Unido
\end{tabular}


Em que:

$E_{p}=$ evapotranspiração de referência $\left(m m\right.$ dia $\left.^{-1}\right)$;

$\mathrm{R}_{\mathrm{n}}=$ radiação líquida na superfície das culturas $\left(\mathrm{MJ} \mathrm{m}^{2} \mathrm{dia}^{-1}\right)$;

$\mathrm{G}=$ fluxo de calor no solo $\left(\mathrm{MJ} \mathrm{m}^{2} \mathrm{dia}^{-1}\right)$;

$\mathrm{T}$ = média diária da temperatura do ar a $2 \mathrm{~m}$ de altura $\left({ }^{\circ} \mathrm{C}\right)$;

$\mathrm{u}_{2}=$ velocidade do vento a $2 \mathrm{~m}$ de altura $\left(\mathrm{m} \mathrm{s}^{-1}\right)$;

$\mathrm{e}_{\mathrm{s}}=$ pressão da saturação de vapor $(\mathrm{kPa})$;

$\mathrm{e}_{\mathrm{a}}=$ pressão de vapor atual $(\mathrm{kPa})$;

$\left(e_{s}-e_{a}\right)=$ déficit de saturação de vapor $(k P a)$;

$\Delta$ = inclinação da curva da pressão de vapor versus

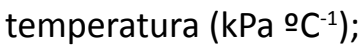

\section{Modelo de regressões}

A primeira etapa do modelo de regressão é a padronização das séries mensais das vazões naturalizadas do ONS, utilizando a Equação 2:

$z_{i, j, k}=\frac{q_{i, j, k}-\overline{q_{i, j}}}{\sigma_{i, j}}$

Em que:

Z = vazão normalizada;

$\mathrm{i}=$ número de meses (variando de 1 a 12);

$\mathrm{k}=$ número de anos (variando de 1931 a 2008);

$\mathrm{j}=$ número de postos utilizados (no total de 188);

$\mathrm{q}_{\mathrm{i}, \mathrm{j}, \mathrm{k}}=$ vazão naturalizada do posto $\mathrm{j}$ no mês i padronizado no ano k;

$\overline{\mathrm{q}_{\mathrm{i}, \mathrm{j}}}=$ matriz que representa a média de todos os meses e postos;

$\sigma_{i, j}=$ matriz que representa o desvio padrão da série mensal de todos os postos.

Após a padronização, é realizada a regressão linear das vazões naturalizadas do ONS e são obtidos os pa-

\section{Análise das projeções}

\section{Cálculo da anomalia média anual}

Para o cálculo da anomalia média anual foi considerada a Equação 4, dada pela diferença entre a média anual do cenário do século XXI e a média anual do cenário histórico sobre a média anual do cenário histórico:
$\gamma\left(\mathrm{kPa} \stackrel{\circ}{ } \mathrm{C}^{-1}\right)=$ constante psicrométrica.

A velocidade do vento em $2 \mathrm{~m}$ de altura, a radiação líquida, a pressão de vapor real ou atual e as temperaturas máxima e mínima formam um conjunto de dados básicos para a estimativa da $\mathrm{E}_{\mathrm{p}}$ via Penman-Monteith. Entretanto, para a estimativa do método, foram utilizadas simplificações físicas; os dados de entrada para esse método foram as temperaturas média, mínima e máxima, enquanto a velocidade do vento a 2 $\mathrm{m}$ de altura foi considerada $2 \mathrm{~m} / \mathrm{s}$. Mais detalhes podem ser encontrados em Silveira et al (2014).

râmetros de cada posto que não possui o SMAP, considerando os demais postos como variáveis explanatórias. A regressão linear é dada pela Equação 3:

$z_{i, p j, k}=\sum_{p k=1}^{p k=21} z_{i, p k, k} \cdot \beta_{i, p k}$

Em que:

pk = bacias cujas vazões são obtidas via SMAP. Portanto, pk varia de 1 a 21 ;

pj = postos que não possuem o SMAP calibrado. Logo, pj varia de 1 a 167;

$\beta \mathrm{i}, \mathrm{pk}=$ coeficientes das regressões.

Com os coeficientes - aferidos a partir das séries de vazões naturalizadas do ONS - e as vazões obtidas via SMAP com dados dos modelos globais do IPCC-AR5, são obtidas as vazões para os cenários de mudança climática para os demais postos utilizando os parâmetros de regressão.

$$
A_{\text {anual }}=\frac{\left(p_{x x \mid}^{u}-p_{x x}^{a}\right)}{p_{x x}^{a}} \cdot 100
$$

Em que:

$\mathrm{P}_{\mathrm{XXI}}^{a}=$ média da vazão anual para o cenário do século XXI; $P_{x x}^{a}=$ média da vazão anual para o cenário histórico. 


\section{Análise de tendência das precipitações anuais}

Para analisar a tendência do século XXI, as séries de vazões anuais dos cenários do século XXI foram padronizadas com base nas características da série do cenário histórico de 1950 a 1999. Essa padronização segue a Equação 5:

$Z=\frac{X_{x x 1}^{J}-\overline{X_{x x}}}{\sigma_{x x}}$
Em que:

$Z$ = vazão do cenário do século XXI padronizada;

$\mathrm{X}_{\mathrm{x} \mathrm{j}}^{\mathrm{j}}=$ vazão anual dos cenários RCP4.5 e RCP8.5 para um ano j;

$\overline{X_{x x}}=$ vazão anual média do cenário histórico na série de 1950 a 1999;

$\sigma_{\mathrm{xx}}=$ desvio padrão da série de vazões anuais do cenário histórico.

A metodologia de avaliação de tendência utilizada foi o método clássico de Mann-Kendall-Sen.

\section{RESULTADOS E DISCUSSÕES}

Os impactos das mudanças climáticas não são uniformemente distribuídos no território brasileiro, porém apontam que, para os cenários RCP4.5 e RCP8.5, há maior possibilidade de aumento das vazões no Setor Sul do país ao longo do século XXI, conforme sugerem as Figuras 2 e 3.

No subsistema norte existe uma leve dispersão espacial entre a resposta das vazões dos postos aos cenários de mudanças climáticas. Os postos mais ao norte mostraram reduções de magnitude inferior aos postos mais ao sul, próximos ao centro-oeste.

Assim como no subsistema norte, o Setor Sudeste/ Centro-Oeste apresenta leves divergências espaciais; as anomalias indicam duas regiões que respondem diferentemente às mudanças climáticas: os aproveitamentos mais ao Centro-Oeste do país e a região litorânea do Sudeste brasileiro. Isso é evidenciado para os modelos Commonwealth Scientific and Industrial Research Organisation - MK-3-6-0 (CSIRO-MK-3-6-0) e Beijing Normal University Earth System Model (BNU-ESM), que apresentam anomalias negativas mais intensas na Região Centro-Oeste do Brasil.

No Setor Nordeste a maioria dos modelos apresenta anomalias negativas para ambos os cenários, sendo o módulo destas superiores para o cenário RCP8.5 - o mais pessimista, segundo o IPCC-AR5 - ,sugerindo que essa região semiárida é extremamente sensível ao aumento das concentrações dos gases de efieito estufa no planeta.

Os modelos BNU-ESM, CSIRO-MK-3-6-0 e Community Earth System Model - Biogeochemical Elemental Cycling (CESM1-BGC) indicam reduções em todo o território brasileiro, exceto no extremo Sul do país, para ambos os cenários, porém mais evidente para o RCP8.5. O Model for Interdisciplinary Research on Climate (MIROC5) sugere aumento superior a $25 \%$ no extremo Sul do país, aumento de menor magnitude na Região Sudeste e anomalias negativas, de módulo superior a 5\%, nas regiões Norte e Nordeste, enquanto o HadGEM2-ES indica anomalia positiva no extremo Sul do país, de magnitude superior a $45 \%$, com grande divergência espacial nas demais regiões. Já o modelo BCC-CSM1-1 apresenta normalidade na maior parte das regiões Sudeste e Norte, com reduções superiores no Nordeste, em módulo a 5\%, e anomalias positivas maiores que 5\% no Sul, no período de 2040 a 2069.

As Figuras 4 e 5 exibem as declividades da tendência dos modelos globais para os cenários RCP4.5 e RCP8.5 para as vazões anuais do SIN, segundo o teste de Mann-Kendall-Sen, no período de 2011 a 2098. Analisando o conjunto de modelos que possuem tendência significativa, considerando todo o SIN, há clara evidência de que o aumento das emissões dos gases deefeito estufa sugere maior impacto na geração de energia do setor elétrico, visto que, na maioria dos casos, o módulo da declividade é sempre maior para o cenário RCP8.5, em detrimento do RCP4.5.

Para a maioria dos postos do SIN não há tendência significativa, indicando que a mudança nos padrões médios pode estar associada à maior frequência de eventos extremos em todo o Brasil. Essa tendência é bem pronunciada apenas para alguns modelos no extremo Sul do país, onde se sinaliza aumento nas vazões.

O modelo CSIRO-MK-3-6-0 aponta tendência negativa nos subsistemas nordeste, norte e parte do sudeste/ centro-oeste e ausência de tendência no Setor Sul para ambos os cenários do IPCC-AR5. O modelo BNU-ESM sinaliza tendência negativa para os setores Norte e Sudeste e ausência de tendência nas demais regiões. 

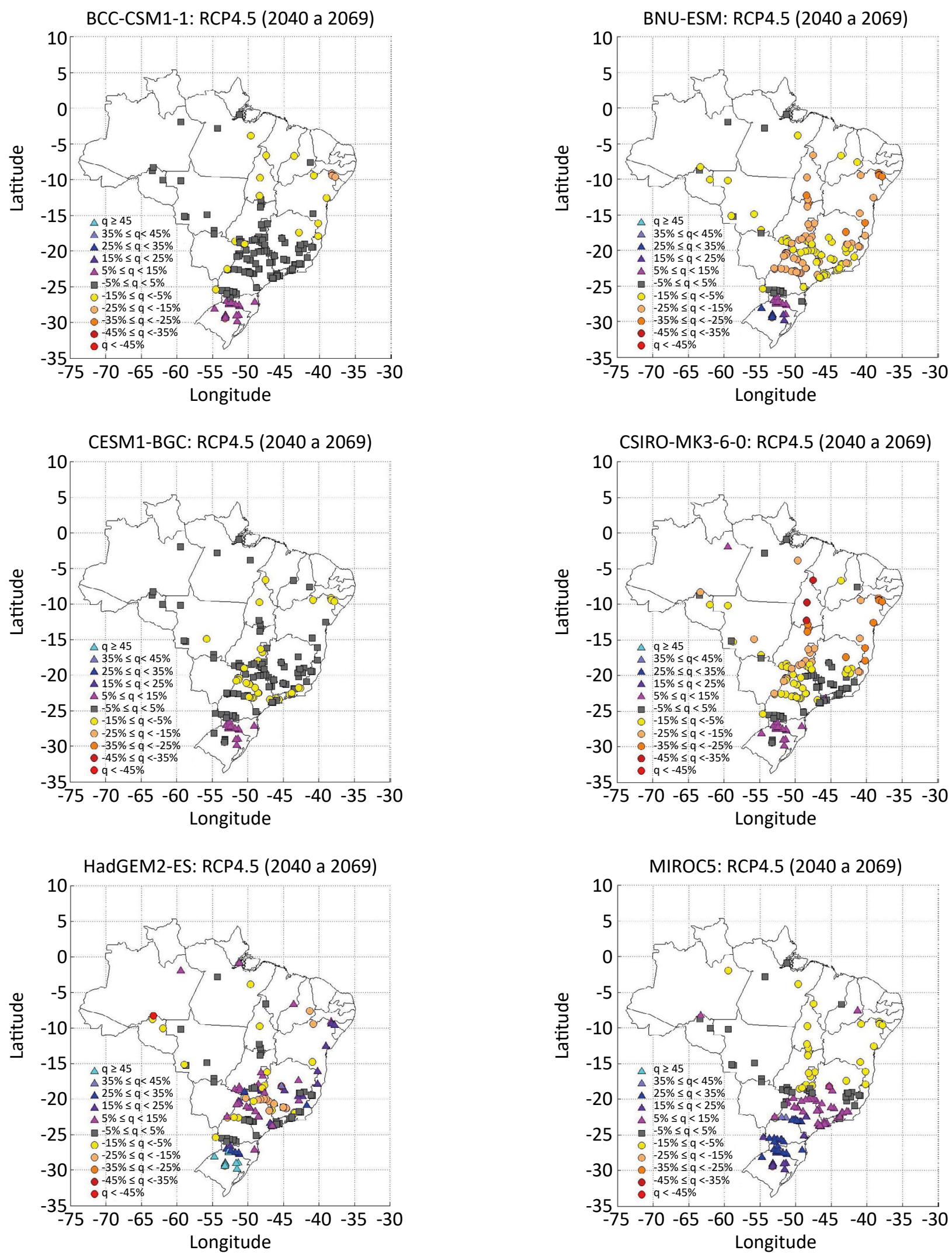

Figura 2 - Anomalias de vazões anuais dos modelos globais do IPCC-AR5 para o período de 2040 a 2069 para o cenário RCP4.5. 

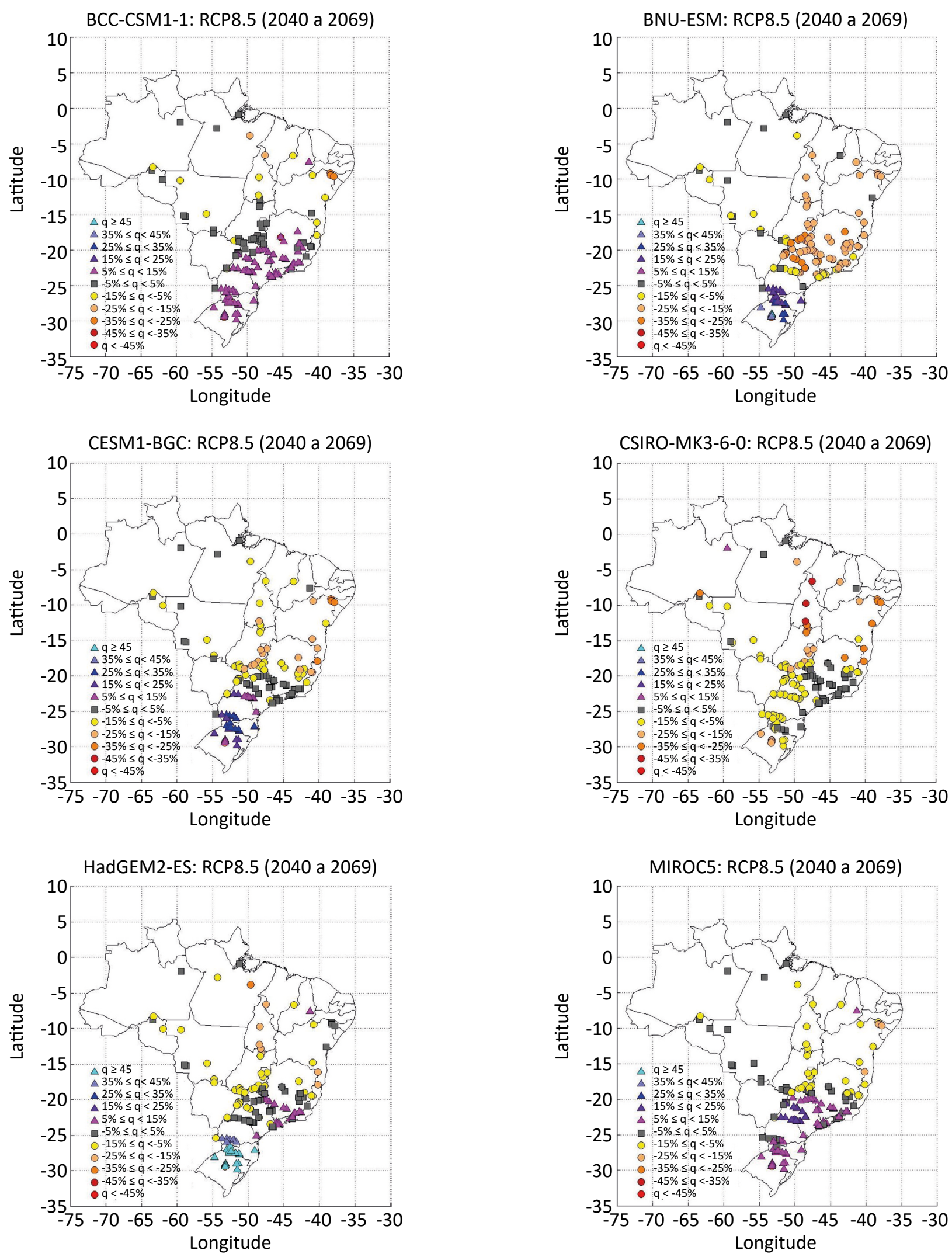

Figura 3 - Anomalias de vazões anuais dos modelos globais do IPCC-AR5 para o período de 2040 a 2069 para o cenário RCP8.5. 

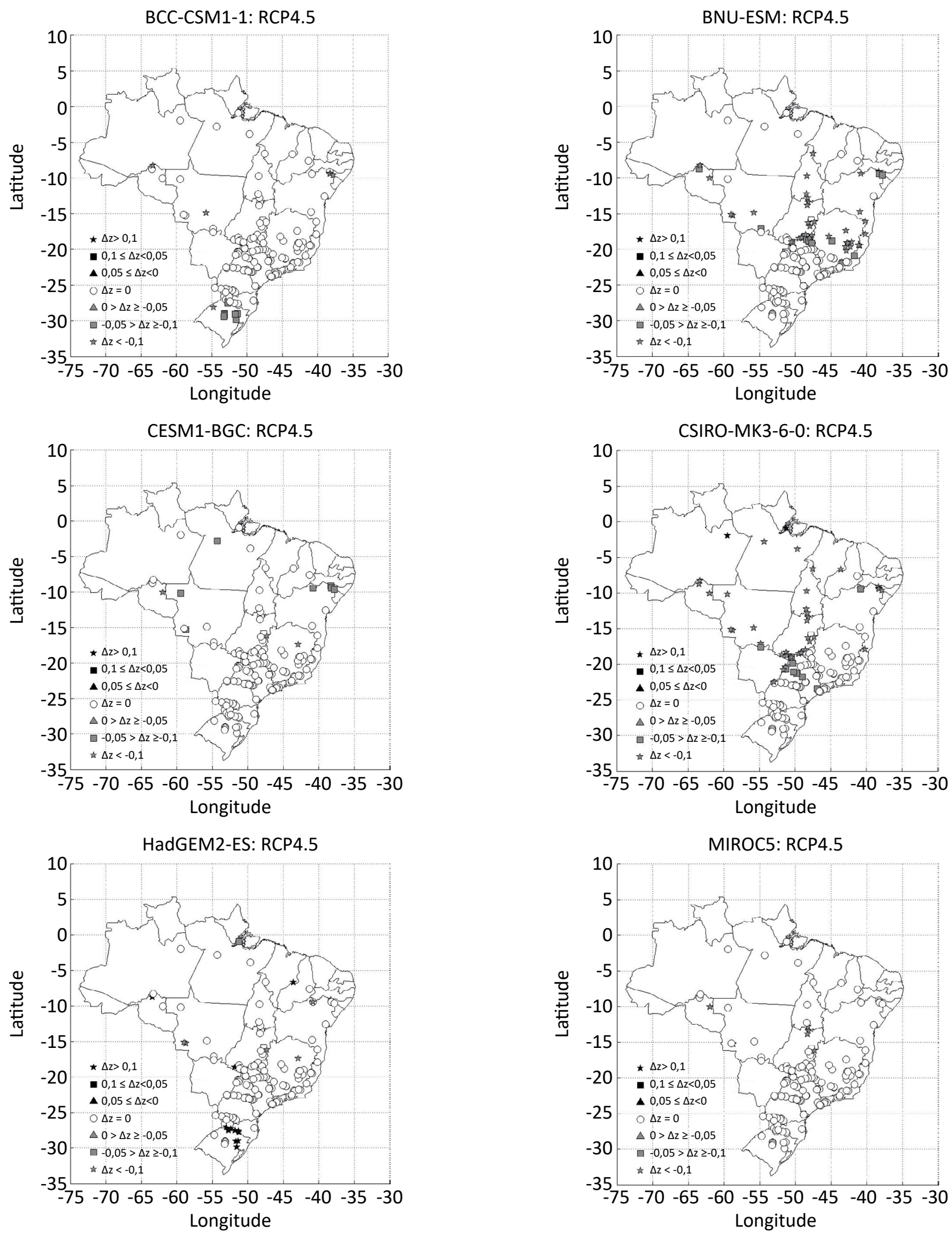

Figura 4 - Tendência, segundo o método de Mann-Kendall-Sen, das vazões médias anuais padronizadas para os modelos do IPCC-AR5 no período de 2011 a 2098 para o cenário RCP4.5. 

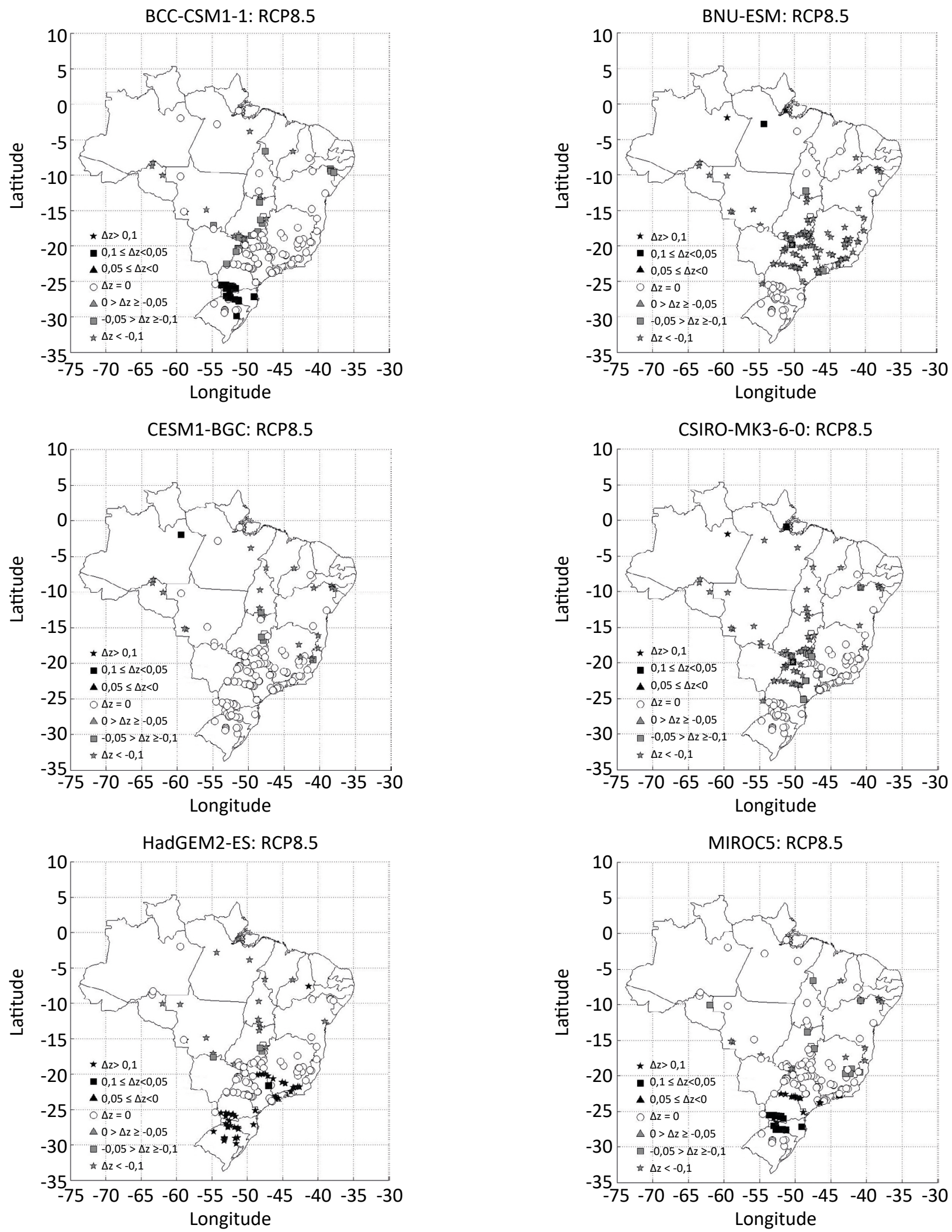

Figura 5 - Tendência, segundo o método de Mann-Kendall-Sen, das vazões médias anuais padronizadas para os modelos do IPCC-AR5 no período de 2011 a 2098 para o cenário RCP8.5. 


\section{CONCLUSÕES}

A análise proposta neste trabalho visou gerar informações acerca do impacto das mudanças climáticas sobre as vazões do setor elétrico do Brasil. Essas informações podem ser usadas pelos gestores na adoção de políticas energéticas e auxiliar medidas que minimizem os impactos de tais cenários.

Os modelos do IPCC-AR5 avaliados aqui apontaram impactos diferentes nas vazões de acordo com os subsistemas do setor hidroelétrico: há maior possibilidade de aumento na Região Sul do país, ao passo que na Região Norte há sinalização de redução. Na Região Nordeste os modelos mostraram maior divergência, indicando incerteza em relação ao impacto da mudança do clima. Na Região Sudeste/Centro-Oeste, apesar da divergência, há maior possibilidade de redução nas vazões.

O aumento das vazões médias anuais no Setor Sul do país e a redução no Setor Nordeste não se refletem na tendência do teste de Mann-Kendall-Sen, sugerindo que as anomalias mostradas podem estar associadas àmaior ocorrência de extremos hidrológicos nas regiões.

Os resultados encontrados para o setor hidroelétrico norte concordam com os obtidos por Lucena et al. (2009), que obtiveram a cenarização da matriz energética do país utilizando informações do IPCC-AR4 e observaram aumento da vulnerabilidade das regiões Norte e Nordeste do Brasil.

A redução da disponibilidade hídrica nas regiões Norte e Sudeste mostrada neste trabalho evidenciou impactos pelo acirramento de conflitos entre usos múltiplos, uma possível desaceleração da economia por causa da redução hídrica para a agricultura e a indústria, bem como o desabastecimento de cidades. Além disso, sinalizou que a expansão do setor elétrico brasileiro para o Setor Norte do país precisa considerar em seu planejamento as mudanças climáticas como elemento de possível redução da oferta da energia na região.

Os impactos das mudanças climáticas somados ao crescimento da demanda energética do Brasil podem levar a uma grande crise no setor energético brasileiro, promovendo investimentos em energias não renováveis em razão do risco de não atendimento dos usuários com a atual matriz energética. Esse tipo de ação pode aumentar o custo da geração de energia e criar um feedback positivo para a mudança climática, bem como intensificar seus efeitos sobre todo o sistema climático e, consequentemente, sobre o país.

As mudanças climáticas representam um desafio para a gestão de recursos hídricos à medida que geram um conjunto de alterações que afetam o ciclo hidrológico. Além dos impactos esperados no regime hidrológico, esperam-se prováveis mudanças na demanda de diversos setores/usuários. A elevação da temperatura e da evapotranspiração poderá acarretar, entre outros efeitos, na maior necessidade de irrigação, refrigeração, consumo humano e dessedentação de animais em determinados períodos e regiões. Essas constatações estão associadas ao estado geral de aumento no consumo de energia, na demanda hídrica da agricultura e do abastecimento humano nos centros urbanos. Portanto, as consequências das mudanças climáticas podem alterar a confiabilidade dos sistemas de água brasileiro atuais e a gestão dos usos e das infraestruturas de suprimento hídrico.

\section{REFERÊNCIAS}

ALLEN, R. G.; PEREIRA, L. S.; RAES, D.; SMITH, M. Crop evapotranspiration: guidelines for computing crop water requirements. Roma: FAOIrrigation and Drainage Paper, 1998.

ALVES, B. C. C.; SOUZA FILHO, F. A.; SILVEIRA, C. S. Análise de tendências e padrões de variação das séries históricas de vazões do Operador Nacional de Sistemas (ONS). Revista Brasileira de Recursos Hídricos, v. 18, n. 4, p. 19-34, 2013. 
ARNELL, N. W. Climate change and global water resources: SRES emission and socioeconomic scenarios. Global Environmental Change, v. 14, p. 31-52, 2004.

BANCOMUNDIAL. Relatório sobre o desenvolvimento mundial de 2010: desenvolvimento e mudança climática. São Paulo: UNESP, 2010.

BATES, B. C.; KUNDZEWICZ, Z. W.; WU, S.; PALUTIKOF, J.P. (Eds.). Climate Change and Water. Technical Paper of the Intergovernmental Panel on Climate Change. Geneva: IPCC, 2008.

BRASIL. Ministério de Minas e Energia (MME). Boletim Mensal de Monitoramento do Sistema Elétrico Brasileiro Abril-2011. Brasília: MME. 2011. 43 p.

CHIEW, F. H. S.; TENG, J.; VAZE, J.; POST, D. A.; PERRAUD, J. M.; KIRONO, D. G. C.; VINEY, N. R. Estimating climate change impact on runoff across southeast Australia: Method, results, and implications of the modeling method, Water Resources Research, v. 45, n. 10, 2009. https://doi.org/10.1029/2008WR007338

INTERGOVERNMENTAL PANEL ON CLIMATE CHANGE (IPCC). Climate change 2001: Impacts, adaptation, and vulnerability. In: MCCARTHY, J.J. Contribution of Working Group II to the Third Assessment Report of the Intergovernmental Panel on Climate Change. Cambridge: Cambridge University Press, 2001. 1032 p.

. Climate Change 2007: Impacts, Adaptation and Vulnerability. Cambridge: IPCC, 2007a. 23 p.

. Climate Change 2007: The physical science basis. Cambridge: IPCC,2007b. 18 p.

.Climate Change 2014: Impacts, Adaptation, and Vulnerability. Part A: Global and Sectoral Aspects. Contribution of Working Group II to the Fifth Assessment Report of the Intergovernmental Panel on Climate Change. Cambridge/ Nova York: Cambridge University Press, 2014. 1132 p.

KROL, M.; JAEGER, A.; BRONSTERT, A.; GÜNTNER, A. Integrated modelling of climate,water, soil, agricultural and socioeconomic processes: A general introduction of the methodologyand some exemplary results from the semi-arid northeast of Brazil. Journal of Hydrology, Amsterdã, v. 328, p. 417-431, 2006.

LOPES, J. E. G.; BRAGA JR., B. P. F.; CONEJO, J. G. L. A. Simulação hidrológica: aplicações de um modelo simplificado. In: SIMPÓSIO BRASILEIRO DE RECURSOS HÍDRICOS, 3., 1981, Fortaleza. Anais... Fortaleza, 1981. p. 42-62.

LUCENA, A. F. P.; SZKLO, A. S.; SCHAEFFER, R.; SOUZA, R. R.; BORBA, B. S. M. C.; COSTA, I. V. L. C.; PEREIRA JÚNIOR, A. O.; CUNHA, S. H. F. The vulnerability of renewable energy to climate change in Brazil. Energy Policy, v. 37, p. 879-889, 2009.

MARENGO, J. A.; SOARES, W. R. Impacto das mudanças climáticas no Brasil e possíveis cenários climáticos: Síntese do Terceiro Relatório do IPCC de 2001. São Paulo: CPTEC-INPE, 2005. 29 p.

MARENGO, J. A.; VALVERDE, M. C. Caracterização do clima no Século XX e Cenário de Mudanças de clima para o Brasil no Século XXI usando os modelos do IPCC-AR4. Revista Multiciência, Campinas, n. 8, maio 2007.

MILLY, P. C. D.; DUNNE, K. A.; VECCHIA, A. V. V. Global pattern of trends in streamflow e water availability in a changing climate. Nature, v. 438, nov. 2005.

NEW, M.; LISTER, D.; HULME, M.; MAKIN, I. A high-resolution data set of surface climate over global land areas. Climate Research, v. 21, p. 1-25, 2002.

NOBRE, C. A. Vulnerabilidade, impactos e adaptação à mudança no clima.In: BRASIL.Presidência da República. Núcleo de Assuntos Estratégicos. Mudança do clima: negociações internacionais sobre a mudança do clima. Brasília, 2005. p. 147-216.

OPERADOR NACIONAL DO SISTEMA (ONS). Atualização de Séries Históricas de Vazões - 1931 a 2010. Brasília: ONS, 2012a. 36 p. 
O Operador Nacional do Sistema elétrico e os procedimentos de rede. Submódulo 9.2 - Recursos hídricos e meteorologia - Acompanhamento, análise e tratamento dos dados hidroenergéticos do Sistema Interligado Nacional. Revisão 1.0. Operador Nacional do Sistema - ONS. Brasília: ONS, 2009. 11 p.

- Programa Mensal da Operação (PMO). Relatório Mensal de Previsão de Vazões e Geração de Cenários de Afluências - Fevereiro/2012. Brasília: ONS, 2012b.

PRADO JR., F. A.; ATHAYDE, S.; MOSSA, J.; BOHLMAN, S.; LEITE, F.; OLIVER-SMITH, A. How much is enough? An integrated examination of energy security, economic growth and climate change related to hydropower expansion in Brazil. Renewable and Sustainable Energy Reviews, v. 53, p.1132-1136, 2016.

SILVEIRA, C. S.; SOUZA FILHO, F. A.; CABRAL, S. L. Análise das projeções de precipitação do IPCC-AR4 para os cenários A1B, A2 e B1 para o século XXI para 28 Nordeste Setentrional do Brasil. Revista Brasileira de Recursos Hídricos, n. 2, p. 117-134, 2013a.

SILVEIRA, C. S.; SOUZA FILHO, F. A.; COSTA, A. A.; CABRAL, S. L. Avaliação de desempenho dos modelos do CMIP5 quanto à representação dos padrões de variação da precipitação no século XX sobre a região Nordeste do Brasil, Amazônia e bacia do Prata e análise das projeções para o cenário RCP8.5. Revista Brasileira de Meteorologia, v. 28, p. 317-330, 2013b.

SILVEIRA, C. S.; SOUZA FILHO, F. A.; LEMOS, W. E. D.; CABRAL, S. L. Mudanças Climáticas e Outorga pelo uso da água no Nordeste do Brasil. In: SOUZA FILHO, F. A.; CAMPOS, J. N. B.; AQUINO, S. H. S. (Orgs.). Gerenciamento de Recursos Hídricos no Semiárido.Fortaleza: Expressão, 2013c. v. 1, p. 243-256.

SILVEIRA, C. S.; SOUZA FILHO, F. A.; LOPES, J. E. G.; BARBOSA, P. S. F.; TIEZZI, R. O. Análise das projeções de vazões nas bacias do setor elétrico brasileiro usando dados do IPCC-AR4 para o século XXI. Revista Brasileira de Recursos Hídricos, v. 19, p. 59-71, 2014.

SILVEIRA, C. S.; SOUZA FILHO, F. A.; SOUZA FILHO, F. A.; CAMPOS, Y. M. L.; COSTA, A. A.; SALES, D. C.; COUTINHO, M. M. Sazonalidade da precipitação sobre o Nordeste Setentrional brasileiro nas simulações do IPCC-AR4. Revista Brasileira de Recursos Hídricos, v. 17, p. 125-134, 2012.

SOITO, J. L. S.; FREITAS, M. A. V. Amazon and the expansion of hydropower in Brazil: vulnerability, impacts and possibilities for adaptation to global climate change. Renewable and Sustainable Energy Reviews, v. 15, p. 3165-3177, 2011.

SOUZA FILHO, F. A. Variabilidade e Mudança Climática nos Semi-Áridos Brasileiros. In: TUCCI, C. E. M.; BRAGA, B. Clima e Recursos Hídricos no Brasil. Porto Alegre: ABRH, 2003. p. 77-116.

SOUZA FILHO, F. A.; PORTO, R. L. L. Acoplamento de Modelo Climáticos e Modelo Hidrológico. In: SIMPÓSIO BRASILEIRO DE RECURSOS HÍDRICOS, 15., 2003, Curitiba. Anais... Porto Alegre: ABRH.

TOMASELLA, J.; RODRIGUES, D. A.; CUARTAS, L. A.; FERREIRA, M.; FERREIRA, J. C.; MARENGO, J. Estudo de impacto das mudanças climáticas sobre os recursos hídricos superficiais e sobre os níveis dos aqüíferos na bacia do rio Tocantins. Convênio de Cooperação Técnico-Científica INPE-VALE. Convênio de Cooperação Técnico-Científica. Manaus: INPE/CCST, 2009.

VAN VLIET, M. T.; WIBERG, D.; LEDUC, S.; RIAHI, K. Power-generation system vulnerability and adaptation to changes in climate and water resources. Nature Climate Change, 2016.

VAN VLIET, M. T.; YEARSLEY, J. R.; LUDWIG, F.; VÖGELE, S.; LETTENMAIER, D. P.; KABAT, P. Vulnerability of US and European electricity supply to climate change. Nature Climate Change, 2012. 